\title{
Infrared observation of Mira variables and their galactic distribution
}

\section{Ryo Miyanoshita, Toshihiro Omodaka, Toshihiro Handa, Tatsuya Kamezaki, Chihiro Nakai, and Hiroshi Fujiwara,}

Depart. Astron. \& Phys., Kagoshima Univ., Korimoto 1-21-35, Kagoshima 890-0065, Japan email: omodaka@sci.kagoshima-u.ac.jp

\begin{abstract}
Since November 2003, we have monitored AGB candidates in infrared wavelengths with the Kagoshima $1 \mathrm{~m}$ telescope. We present the latest results based on the data until June 2011. For each of 400 monitored AGB stars, we estimate the periods, amplitudes, and mean apparent magnitudes in $\mathrm{K}$ band. Based on the two color diagram we choose 259 objects as Miras. We use the period-luminosity relation to obtain distances to them. As results we show the 3 dimensional distribution of 259 Mira variables.
\end{abstract}

Keywords. stars: AGB — stars: distances — Galaxy: structure

We obtained monitoring observations of AGB stars in $\mathrm{J}, \mathrm{H}$, and $\mathrm{K}$ bands with the Kagoshima $1 \mathrm{~m}$ telescope. In the monitored targets, we chose 259 objects as Mira variables and obtain their 3 dimensional distribution using the period-luminosity relation calibrated with annual parallaxes as observed using VERA (Nakagawa et al. 2012).

In the Face-on distribution (Fig. 1A), there are some hints of concentration along the Orion and Sagittarius Arms. The Edge-on distribution (Fig. 1B) shows that $90 \%$ of Mira variables are confined within the $1 \mathrm{kpc}$ from the Galactic plane. The scale height was estimated to be $0.45 \mathrm{kpc}$ which suggests that the Mira variables surely belong to the thick disk.
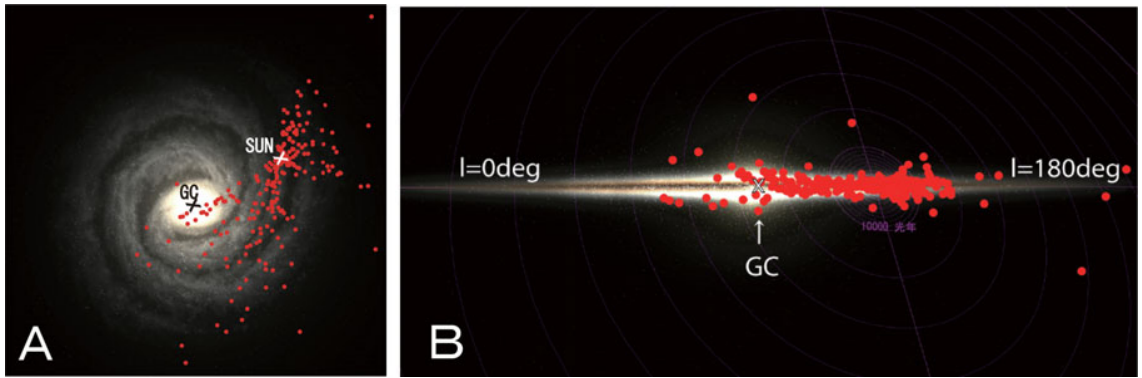

Figure 1. Galactic distribution of 259 Mira variables. Panel A is the face-on view. Panel B is the edge-on view.

\section{References}

Matsunaga, Noriyuki et al. 2009, Mon. Not. R. Astron. Soc., 399, 1709-1729

Nakagawa, Akiharu et al. 2013, in: R. de Grijs \& G. Bono (eds.) "Advancing the Physics of Cosmic Distances" Proc. IAU Symp. 289, in press

Nishiyama, Shogo et al. 2006, The Astrophysical Journal, 638, 839-846

van der Veen \& H. J. Habing. 1988, Astron. Astrophys, 194, 125-134

Whitelock, Patricia et al. 2000, Mon. Not. R. Astron. Soc., 319, 728-758 